\title{
Query Expansion Using Term Distribution and Term Association
}

\author{
Dipasree Pal \\ Information Retrieval Society of India \\ Kolkata, West Bengal, India \\ dipasree.pal@gmail.com
}

\author{
Mandar Mitra \\ Indian Statistical Institute \\ Kolkata, West Bengal, India \\ mandar@isical.ac.in
}

\author{
Samar Bhattacharya \\ Jadavpur University \\ Kolkata, West Bengal, India \\ samar.bhattacharyya@gmail.com
}

\begin{abstract}
Good term selection is an important issue for an automatic query expansion (AQE) technique. AQE techniques that select expansion terms from the target corpus usually do so in one of two ways. Distribution based term selection methods typically compute the divergence between the distribution of a term in the (pseudo) relevant documents with that in the whole corpus (or a random distribution). Association based term selection, on the other hand, uses information about how a candidate term co-occurs with the original query terms. Our goal in this study is to investigate how these two classes of methods may be combined to improve retrieval effectiveness. We propose the following combination-based approach. Candidate expansion terms are first obtained using a distribution based method. This set is then refined based on the strength of the association of terms with the original query terms. We test our methods on several TREC collections. The proposed combinations generally yield better results than each individual method, and are comparable to AQE approaches such as RM3. En route to our primary goal, we also propose some modifications to an existing AQE method which lead to improved performance.
\end{abstract}

\section{KEYWORDS}

Query Expansion, Term Distribution, Term Association

\section{INTRODUCTION}

The well-known vocabulary mismatch problem is one significant factor that makes searching difficult. A user's query $Q$ and a useful item $D$ in a database (henceforth referred to as a document) may use different vocabulary to refer to the same concept. Retrieval systems that rely on keyword-matching may not detect a match between $Q$ and $D$. A good retrieval system must bridge the vocabulary gap that exists between useful documents and the user's query. Query Expansion (QE), the addition of related terms (called expansion terms) to a user's query, is one important technique that attempts to solve this problem by increasing the likelihood of a match between the query and relevant documents.

A wide variety of methods for Automatic Query Expansion (AQE) have been proposed over the last 15-20 years. These methods find related terms from different sources such as the target corpus, linguistic resources like Wordnet [13], thesauri [20], ontologies [5], the World Wide Web, Wikipedia [32] and query logs [33]. A recent

Permission to make digital or hard copies of part or all of this work for personal or classroom use is granted without fee provided that copies are not made or distributed for profit or commercial advantage and that copies bear this notice and the full citation on the first page. Copyrights for third-party components of this work must be honored. For all other uses, contact the owner/author(s).

The Second Women in Data Science (WinDS) Workshop, co-located with The Web Conference 2018, April 24, 2018, Lyon, France.

(c) 2018 Copyright held by the owner/author(s)

https://doi.org/10.18122/winds/2/boisestate

\begin{tabular}{ccccc}
\hline Baseline & $\begin{array}{c}\text { Assoc. based } \\
(\text { method })\end{array}$ & $\begin{array}{c}\text { Distr. based } \\
(\operatorname{method} D)\end{array}$ & Max \\
\hline MAP & 0.218 & $0.252(+15.5 \%)$ & $0.257(+18.0 \%)$ & $0.291(+33.5 \%)$ \\
Better on & - & 78 queries & 91 queries & 100 queries \\
\hline
\end{tabular}

Table 1: Potential improvement obtainable in principle by judiciously choosing QE techniques

survey of such techniques can be found in [9]. Of all these techniques, methods that use the target corpus as a source of expansion terms are among the most widely used because they are simple and require no additional resources.

Many target-corpus-based AQE techniques can be broadly classified into one of two groups: distribution based and association based. Distribution based methods select terms by comparing the distribution of a term in the (pseudo) relevant documents with its distribution in the whole corpus. This is typically done by computing a divergence measure between the two distributions. Broadly, such methods select terms that are more likely to occur in the (pseudo) relevant documents than in a document chosen randomly from the entire corpus. On the other hand, association based methods select expansion terms on the basis of their association (or co-occurrence) with all query terms. A term that tends to co-occur with all / many of the query terms is regarded as a good expansion term.

While a number of distribution / association-based QE techniques have been shown to be effective on average (i.e. when their overall performance across a large set of queries is measured), the impact of different $\mathrm{QE}$ techniques on individual queries can vary greatly. A study by Billerbeck and Zobel [6] focuses on the related question of per-query parameter choices for a particular $\mathrm{AQE}$ method. Using TREC disks 4 and 5, and the title field of queries 401-450 (please see Section 4 for more details), the authors show that the best parameter choices per query vary wildly. They conclude that "individually tuning parameters to queries can give much better performance than use of fixed values", but it is not clear how such tuning could be done.

Our preliminary experiments with TREC queries 301-450 lead us to a similar conclusion with regard to AQE paradigms (rather than parameters). Table 1 shows the Mean Average Precision (MAP) scores for three retrieval methods: a baseline strategy that uses original, unexpanded queries, and representative distribution-based and association-based QE methods (described in Sections 3.1.1 and A.1 respectively). The QE methods are significantly superior to the baseline on average, but the improvements are observed only on 78 (for method A) and 91 (for method D) queries. For almost all the remaining queries, these methods actually hurt performance.

Figure 1 graphically compares the performance of these two methods. Points on the X-axis correspond to queries; for each query, the difference in performance (AP) between $\mathrm{D}$ and $\mathrm{A}$ is shown on the $\mathrm{Y}$-axis (thus, bars above the $\mathrm{X}$-axis correspond to queries for which D performs better). From the figure, it is clear that each of 


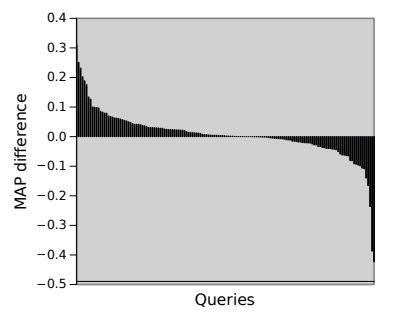

Figure 1: Difference in AP for method D and method A on TREC queries 301-450.

these methods outperforms the other on about half the queries used in this experiment, even though the overall performance figures for these two QE methods are comparable.

Based on this observation, we hypothesise that if we combine these two approaches by considering both distribution information and association information, we should be able to improve overall performance. In this study, therefore, we investigate the possibility of improving retrieval effectiveness by combining association- and distribution-based QE approaches. We first select representative methods from each category, viz. LCAmod ${ }^{1}$, RM3 [1] (associationbased), and KLD [8], Bo1 [2] (distribution-based). Two methods - one from each category - are then combined. Various ways of combining the two methods were explored. The following approach seems to work well: we select a relatively large number of candidate expansion terms using the distribution based method. Some of these are filtered out using information from the association based method. The refined set is finally used for query expansion.

We test our combined method on several TREC collections. Our proposed method yields significant improvements on all collections over a baseline that uses the original, unexpanded queries. More importantly, the combined methods yield improvements over the individual AQE methods for most of the collections. Additionally, such a combination is comparable to widely used QE methods, like those proposed in $[1,2]$.

In the next section, we discuss the relationship between this study and related work. Section 4 briefly describes the IR system and the test collections used in our experiments. Section 3 reviews the existing AQE methods that are used in this study, as well as the proposed method for combining AQE techniques. Results comparing the proposed methods with existing ones are presented in Section 5. Finally, Section 6 summarizes some related issues that need to be studied in future work.

\section{RELATED WORK}

Research on automatic query expansion dates back to the 1960 s. Rocchio's relevance feedback method [26] is still used in its original and modified forms for AQE. The availability of the TREC collections, and the widespread success of AQE on these collections stimulated further research in this area. Carpineto and Romano [9] provide a recent and comprehensive survey of $A Q E$ techniques. We primarily focus here on AQE techniques that are either associationor distribution-based. Finally, we discuss earlier work that investigates combination-based AQE methods.

\footnotetext{
${ }^{1}$ Based on LCA [31], and described in more detail in Appendix A.
}

Association-based QE techniques Early work on associationbased AQE includes "concept-based" QE [23] and phrasefinder [14]. Both methods make use of term co-occurrence information extracted from a corpus. Local context analysis (LCA) [30, 31] is another well-known method that selects expansion terms based on whether they have a high degree of co-occurrence with all query terms. However, in LCA, co-occurrence information is obtained from a set of top-ranked documents retrieved in response to the original query, rather than the whole target corpus.

Relevance-based language models [16] constitute another, more recent, co-occurrence based approach that performs well. This method is based on the Language Modelling framework. The query and relevant documents are all assumed to be generated from an underlying relevance model. This model is estimated based on (only) the pseudo relevant documents for a particular query. This approach was subsequently refined by Abdul-Jaleel et al. [1]. The refinement, called RM3, incorporates the original query when estimating the relevance model. According to a comparative study by Lv and Zhai [17], RM3 is the most effective and robust among a number of state-of-the-art AQE methods.

Wu et al. [29] use a split-list approach for feedback. For each query term / phrase, representative contexts are collected from a few top-ranked documents. These contexts are then ranked. Each document gets a weight that is obtained by summing up the weights coming from only a few, top-ranked contexts. The main difference between this and other work discussed here is that the method makes use of actual (as opposed to pseudo) relevance information.

Distribution-based QE techniques As early as 1978, Doszkocs [12] proposed the interactive use of an associative dictionary that was constructed based on a comparative analysis of term distributions. Also well known is Robertson's analysis of term selection for query expansion [25]. More recently, Carpineto et al. [8] proposed an effective QE method based on information theoretic principles. This method computes the Kullback-Leibler divergence (KLD) between the probability distributions of terms in the relevant (or pseudo-relevant) documents and in the complete corpus. Amati [2] proposes a new distribution based method which uses Bose-Einstein statistics. This method also calculates the divergence between the distribution of terms in the pseudo relevant document set and a random distribution.

While Lavrenko and Croft [16] incorporated feedback within a language modelling framework by using the overall association between an expansion term and the original query terms, Zhai and Lafferty [34] achieve the same objective using two different approaches that are more distribution based in spirit. One method is based on a generative probabilistic model of feedback documents. The other tries to construct a model that minimises its own aggregated KL-divergence with the feedback documents.

Combination-based techniques Efforts have also been made to combine AQE methods in various ways to improve retrieval effectiveness. Vechtomova et al. [28] construct an expanded query by adding the collocates (terms within close proximity) of each query word. This expanded query is combined with another expanded query that is constructed using Okapi relevance feedback [15]. Sun et al. [27] introduce a combination based method that uses a modified version of LCA. Each sentence of each snippet returned by 
Google in response to a query is regarded as a passage. The snippets are parsed. LCA-based association information is combined with the dependency relations extracted from these parse trees. The disadvantage to this approach is that it uses the Google Search API, a black box component that changes over time. Carpineto et al. [10] combined the scoring functions of a number of methods, all of them distribution-based, to obtain improvements. In contrast, we combine a distribution-based method with an association-based method (based on our belief that these two classes of methods offer different advantages). Also, rather than combining scores, we use one method to refine the set of terms selected by the other. More recently, Lv et al. [18] propose a boosting-based learning algorithm to improve PRF by optimally combining a set of basis feedback algorithms.

Our approach is somewhat similar in spirit to a method proposed by Cao et al. [7], in which terms selected using standard pseudo relevance feedback (PRF) are refined using a classifier that is trained to differentiate between useful and harmful candidate expansion terms. The method yields improvements over their (relatively low) baseline results. However, the authors note that this method only works well within a supervised learning framework; an unsupervised learning method cannot improve retrieval effectiveness by a large margin. This is an important difference between this method and our proposed method.

Our work is most strongly related to that of Pérez-Agüera and Araujo [21], who also combine co-occurrence-based and distributionbased methods. Word co-occurrence is measured using the Tanimoto coefficient. Distributional differences are measured based on KLD or Bose-Einstein statistics. The combination is relatively straightforward: one method is used for term selection and the other for weighting. When both methods are in agreement, no additional benefits are likely to accrue, but when they disagree, the effects of good term selection may be nullified by relatively poor term weight computation. Thus, even though the authors reported positive results on a relatively small Spanish dataset, our implementation of this method did not yield promising results on various TREC datasets (described in the next section). We therefore used the well-known LCA and RM3 methods (instead of Tanimoto coefficient) to quantify term association. Also, instead of simply using one method for term selection and the other for weighting, we combined both methods for selection.

\section{METHODS}

\subsection{Basic methods}

We first review KLD, Bo1, LCA and RM3, the existing methods that form the base of our approach. These were chosen as representative because they (1) are well-known, highly cited, and / or widely used as a basis for comparison in QE-related research; and (2) perform comparatively better than other methods in these categories (including recently proposed ones).

3.1.1 Distribution based method I: KLD. The approach proposed by Carpineto et al. [8] is one of the two distribution based term ranking methods used in this study. In this method, all terms in the pseudo relevant set are treated as candidate expansion terms. Let $P R D$ and $C$ represent the (pseudo) relevant documents (PRD) and the whole corpus respectively. We use $p_{r}$ and $p_{c}$ to denote the unigram probability distribution of terms in $P R D$ and $C$ respectively; $p_{r}$ and $p_{c}$ are calculated as shown in Equations 1 and $2(t f(t, d)$ represents the term frequency of term $t$ in document $d$ ).

$$
\begin{gathered}
p_{r}(t)=\frac{\sum_{d \in P R D} t f(t, d)}{\sum_{d \in P R D} \sum_{t^{\prime} \in d} t f\left(t^{\prime}, d\right)} \\
p_{c}(t)=\frac{\sum_{d \in C} t f(t, d)}{\sum_{d \in C} \sum_{t^{\prime} \in d} t f\left(t^{\prime}, d\right)}
\end{gathered}
$$

The contribution of a term to the divergence between $p_{r}$ and $p_{c}$ is given by Equation 3. Terms for which this contribution is the largest are selected as expansion terms.

$$
S(t)=p_{r}(t) * \log \frac{p_{r}(t)}{p_{c}(t)}
$$

$S(t)$ is also used as the term weight of a candidate expansion term $t$.

In our experiments with KLD (and other methods), we use Equations 4 to 6 to merge the original query terms with the candidate expansion terms to formulate the final expanded query. The weights of original query terms are normalized using the maximum original query term weight (Eqn. 4); weights of expansion terms are similarly normalized (Eqn. 5). These weights are combined via a parameter $\lambda$ to obtain the final weight of a term $t$ in the expanded query (Eqn. 6).

$$
\begin{aligned}
& \text { score }_{\text {orig }}(t)=\frac{1+\log (t f(t, Q))}{1+\max _{t^{\prime} \in Q} \log \left(t f\left(t^{\prime}, Q\right)\right)} \\
& \text { score }_{\exp }(t)=\frac{S(t)}{\max _{t^{\prime} \in d \in P R D} S\left(t^{\prime}\right)} \\
& \text { score }(t)=\lambda \times \text { score }_{\text {orig }}(t)+(1-\lambda) \times \text { score }_{\exp }(t)
\end{aligned}
$$

3.1.2 Distribution based method II: Bo1. The second, more recent, distribution based term ranking model we considered is Bo1, which is the most effective variant of the Divergence From Randomness (DFR) term weighting model [19, 22]. In this model based on Bose-Einstein statistics, the informativeness of a term $t$ is measured by the divergence between its distribution in the top ranked documents and a random distribution. Specifically, the score of a candidate expansion term $t$ is given by

$S(t)=\left(\sum_{d \in P R D} t f(t, d)\right) * \log _{2}\left(\frac{1+t f_{a v g}(t, C)}{t f_{a v g}(t, C)}\right)+\log _{2}\left(1+t f_{a v g}(t, C)\right)$

where

$$
t f_{a v g}(t, C)=\sum_{d \in C} t f(t, d) / N
$$

denotes the average term frequency of $t$ in the collection; $N$ is the number of documents in the collection. As in Section 3.1.1, we use Equations 4-6 to merge the original query with the expansion terms and formulate the new expanded query. 
3.1.3 Association based method I: LCAmod. Equations 9-12 define our approach for calculating the association between a candidate term $t$ and the query $Q$ (in these equations, $N_{t}$ stands for the number of documents containing $t, P R D$ denotes the set of pseudorelevant documents, and $\delta$ is set to 0.1 as suggested by Xu and Croft [31]). The motivation and experimental results pertaining to this formulation are described more fully in Appendix A.

$$
\begin{gathered}
i d f_{t}=\log _{10} \frac{N-N_{t}+0.5}{N_{t}+0.5} \\
c o\left(t, q_{i}\right)=\sum_{d \in P R D}\left(\min \left(t f(t, d), t f\left(q_{i}, d\right)\right) *\right. \\
\left.\max \left(i d f_{t \vee q_{i}}, 0\right) * \frac{\operatorname{Sim}(d, Q)}{\max _{d^{\prime} \in P R D} \operatorname{Sim}\left(d^{\prime}, Q\right)}\right)
\end{gathered}
$$

Here $i d f_{t \vee q_{i}}$ denotes the idf of term $t$ or $q_{i}$, whichever has the lower term frequency in document $d$.

$$
\begin{gathered}
\text { codegree }\left(t, q_{i}\right)=\frac{\log _{10}\left(\operatorname{co}\left(t, q_{i}\right)+1\right)}{\log _{10}|P R D|} \\
S(t)=\sum_{i=1}^{k} i d f_{q_{i}} * \log _{10}\left(\delta+\operatorname{codegree}\left(t, q_{i}\right)\right)
\end{gathered}
$$

As before, the $T$ terms with the highest association scores $(S(t))$ are selected as expansion terms. The final term weights in the expanded query are determined using Equations 4 to 6.

3.1.4 Association based method II: RM3. Relevance-based language models $[1,16]$ constitute a more recent association based approach. In this approach, the association $S(t)$ between a word $t$ and a query $Q=q_{1}, \ldots, q_{k}$ can be measured by $P\left(t, q_{1}, \ldots, q_{k}\right)$, the joint probability of observing the word together with the query words, when these words are all sampled from an (unknown) relevance model. This relevance model is estimated from a finite universe $\mathcal{M}$ of unigram distributions each of which corresponds to a (pseudo) relevant document. Under the assumption that $t, q_{1}, \ldots, q_{k}$ are independently and identically sampled from $M \in \mathcal{M}$,

$$
\begin{aligned}
S(t) & =P\left(t, q_{1}, \ldots, q_{k}\right) \\
& =\sum_{M \in \mathcal{M}} P(M) P(t \mid M) \prod_{i=1}^{k} P\left(q_{i} \mid M\right) \\
& =\frac{1}{\# P R D} \sum_{d \in P R D}\left(\frac{t f(t, d)}{|d|} \times \prod_{i=1}^{k} \frac{t f\left(q_{i}, d\right)+\mu P\left(q_{i} \mid C\right)}{|d|+\mu}\right)_{13)}
\end{aligned}
$$

where $\mu=2500$ is a smoothing parameter, and $P\left(q_{i} \mid C\right)=p_{c}\left(q_{i}\right)$. This implementation is analagous to the Lemur ${ }^{2}$ RM3 method. However, we used the i.i.d. sampling approach instead of the conditional sampling method recommended in [16], since this gave us better results during initial experiments with the TREC678 dataset. Equations 14 to 16 show, as before, how the expanded query terms are added to the original query.

$$
\text { score }_{\exp }(t)=\frac{S(t)}{\sum_{d \in P R D} \sum_{t^{\prime} \in d} S\left(t^{\prime}\right)}
$$

\footnotetext{
${ }^{2} \mathrm{http}: / /$ www.lemurproject.org/
}

score $_{\text {orig }}(t)=\frac{t f(t, Q)}{|Q|}$
score $(t)=\lambda *$ score $_{\text {orig }}(t)+(1-\lambda) *$ score $_{\text {exp }}(t)$ where $0 \leq \lambda \leq 1$

\subsection{Combining association based method with distribution based method}

Section 3.1 reviews two different types of query expansion methods. In this section, we describe a hybrid approach that combines the above methods to improve retrieval effectiveness.

We conducted some preliminary experiments to explore various ways to combine individual methods. Our first attempt involved simply adding up the normalized weights of the expansion terms as computed by the individual methods. This combination only rarely yields significant improvements. For most collections, the combination is either worse than the superior individual method, or achieves only marginal improvements that are not statistically significant. It appears that this particular combination results in decreasing the importance of the original query terms, and gives higher weights to the expansion terms. This leads to degraded performance.

On the other hand, combining weights by multiplying them together appears to be excessively conservative, i.e., the combination weight of an expansion term tends towards the lower of the two weights. For many queries, the benefits of expansion are thereby lost. Thus, this approach also turns out not to work very well.

Next, we tried to apply the methods sequentially: the original query is expanded using one of the methods, and the expanded query is then used as the initial query for the other method and expanded further. Both combinations were tried: KLD first, followed by LCA (LCA-over-KLD), and vice versa (KLD-over-LCA). Our experiments show that LCA-over-KLD is worse than KLD-overLCA; in turn, KLD-over-LCA is worse than KLD.

The final approach that we tried also applies the methods sequentially, but in a different way. One of the methods is used first to create a large expanded query. This query is then refined (instead of being expanded further) using the other method. This method turns out to work well, and yields significant improvements over the individual methods.

We arbitrarily choose a distribution-based method - KLD (Equation 3) or Bo1 (Equation 7) - to first select (and weight) a relatively large number of candidate terms that occur preferentially in a few top-ranked documents, where the proportion of relevant documents is expected to be high. This set is then refined using co-occurrence information: terms that do not co-occur significantly with original query terms are discarded. Conversely, candidate expansion terms that are relatively poorly ranked by the distribution-based method have a chance to be included in the final query if they adequately co-occur with the original query terms. More precisely, the candidate terms are re-ranked using an association-based method LCAmod (Equation 12) or RM3 (Equation 13) - that looks at a larger number of top-ranked documents. The top $T$ terms from this re-ranked list are chosen as the final expansion terms. However, we retain the weights of these terms as determined by the distribution based method. As before, the final term weights in the expanded query are determined using Equations 4 to 6. 


\begin{tabular}{ccc}
\hline Coll. Id. & \# of queries & Documents \\
\hline TREC123 (Q51-Q200) & 150 & TREC disks 1, 2 \\
\hline TREC4 (Q202-Q250) & 49 & TREC disks 2, 3 \\
\hline TREC5 (Q251-Q300) & 50 & TREC disks 2, 4 \\
\hline TREC678 (Q301-Q450) & 150 & TREC disks 4, 5 - CR \\
\hline ROBnew (Q601-Q700) & 100 & TREC disks 4, 5 - CR \\
\hline TREC910 (Q451-Q550) & 100 & WT10G \\
\hline GOV2 (Q701-Q850) & 150 & GOV2 \\
\hline
\end{tabular}

Table 2: Test collections

\section{EXPERIMENTAL SETUP}

Table 2 lists the details of the test collections used in our experiments. As real-life queries are very short, we used only the title field of all these queries, except for the TREC4 queries, which contain only the description field. Many of the queries thus contain only one term, and most of the remainder are no longer than three words; only the TREC4 queries are longer.

We used the Terrier ${ }^{3}$ retrieval system for our experiments. At the time of indexing, stopwords are removed and Porter's stemmer is used as preprocessing. All documents and queries are indexed using single terms, no phrases are used. The IFB2 variant of the Divergence From Randomness model [3] is used for term-weighting in all our experiments. We found IFB2 performs better compared to the other DFR variants available within Terrier. Parameters are set to the default values used in Terrier. Table 3 shows the impact of tuning the two parameters that are important in target corpus based AQE methods, that is $D$, the number of pseudo-relevant documents, and $T$, the number of expansion terms.

Results are evaluated using standard evaluation metrics: Mean Average Precision (MAP), precision at 10 (P@10), overall recall (number of relevant documents retrieved), and robustness index (RI) [11] with respect to the baseline (no feedback).

\section{EXPERIMENTAL RESULTS}

We now present experimental results for the QE methods described in Section 3. The labels, we use in tables / figures are: KLD(Section 3.1.1), Bo1(Section 3.1.2), LCA(Section A.1), LCAmod(Section 3.1.3), RM3(Section 3.1.4) and KLDLCA, KLDRM3, Bo1LCA, Bo1RM3 (these 4 detaied in Section 3.2). In subsequent tables, results that are statistically significantly better (as determined by a two-tailed paired $t$-test with a confidence level of 95\%) than the baseline (no feedback), KLD, Bo1, LCAmod and RM3 are marked with the superscripts B, $\mathrm{k}, \mathrm{b}, \mathrm{l}$ and $\mathrm{r}$ respectively.

As explained in Section 3.2, in the combination based approach, we first select a large set of candidate terms $(T=100)$ from $D=10$ documents using a distribution-based QE method. The association of these candidate terms with the query terms is computed using the top $D^{\prime}=50$ documents ${ }^{4}$, and the best $T^{\prime}=40$ terms (as determined by an association-based method) are included in the final query. We report results for a total of $2 \times 2=4$ combinations: KLDLCA (LCAmod with KLD), KLDRM3 (RM3 with KLD), Bo1LCA (LCAmod with Bo1), and Bo1RM3 (RM3 with Bo1).

Baselines. We compare the combination-based methods with the following baselines.

(1) No feedback. The original, unexpanded queries are used for retrieval using the baseline method described in Section 4.

\footnotetext{
$\overline{{ }^{3} \mathrm{http}: / / \text { terrier.org/ }}$

${ }^{4}$ Measuring association scores over the top 30-50 documents works about equally well.
}
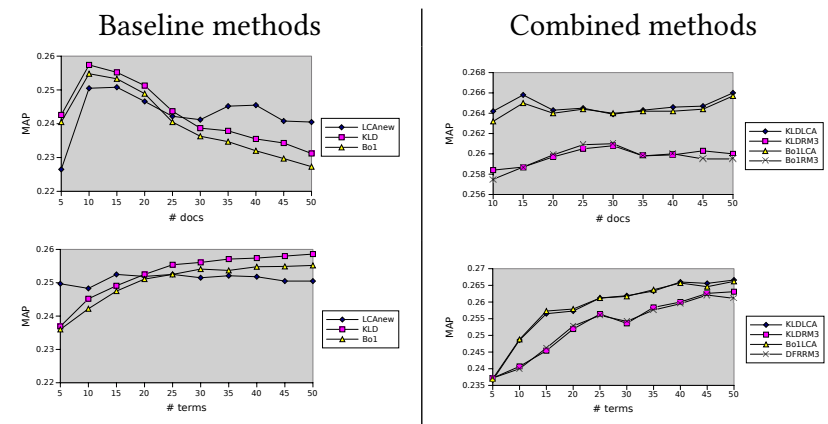

Table 3: Performance (MAP) variations: when $T$ is fixed, and $D$ vary(1st row) and when $D$ is fixed, and $T$ vary(2nd row)

(2) KLD. We find that a setting of $D=10$ top-ranked documents and $T=40$ expansion terms works well for KLD across collections. This is in agreement with the observations of Carpineto et al. [8]. Note that the results presented here correspond to our implementation of KLD within TERRIER. While our implementation provides better results than TERRIER's native implementation of KLD, we were not able to exactly replicate the results reported in [8]. This is likely due to differences between the retrieval functions, indexing or query processing. For example, using full queries (title, desc and narr) on the TREC 8 collection, and BM25 as the base term-weighting formula, we get MAP scores of 0.2992 for KLD (compared to a baseline of 0.2625 ). When using the IFB2 model, however, the baseline is higher (MAP $=0.2753)$, but KLD appears less effective $(\mathrm{MAP}=0.2850)$.

(3) Bo1. For this method, Amati [2] suggested adding $T=10 \mathrm{ex}-$ pansion terms from the top $D=3$ documents. We use $T=40$ and $D=10$ instead, since we wanted a larger number of candidate terms, particularly for the combination-based method. Our experiments confirm that we get comparable results with these parameter settings.

(4) RM3. It is well known that parameter settings can have a major impact on the performance of RM3. We therefore varied the three main parameters, $D, T$ and the interpolation parameter $\lambda$, over the following ranges: $D \in[10,80], T \in[10,100]$, both in steps of 10 , and $\lambda \in[0.1,0.9]$ in steps of 0.1 . However, to avoid running a total of $8 \times 10 \times 9=720$ runs for each collection (particularly GOV2), we tuned the RM3 parameters on TREC678 for the newswire collections (finally choosing $\lambda=0.1, D=10, T=60$ ) and tuned them separately on WT10g, choosing $\lambda=0.2, D=10, T=50$ for the two Web collections (WT10g, GOV2). For a number of datasets, our results for RM3 are superior to those reported in other recent papers ([4], for example).

Table 4 shows that the proposed combined approaches are statistically significantly better than the no-feedback method across all test collections except for TREC5 and TREC910. More importantly, the combined methods generally work better than KLD, Bo1 and LCAmod. Specifically, KLDLCA does better than KLD or LCAmod alone on all measures, and across all datasets. For 6 out of the 7 collections, the combination yields significant improvements in MAP over KLD or LCAmod or both. It is interesting to note that for the sixth collection (TREC5), LCAmod results in a drop in performance compared to the no-expansion baseline. However, the 


\begin{tabular}{|c|c|c|c|c|c|c|c|c|c|c|}
\hline Dataset & Measure & Baseline & KLD & Bo1 & LCAmod & RM3 & KLDLCA & KLDRM3 & Bo1LCA & Bo1RM3 \\
\hline \multirow[t]{5}{*}{ TREC123 } & MAP & 0.218 & $\begin{array}{l}0.274 \\
(25.4)\end{array}$ & $\begin{array}{l}0.272 \\
(24.4)\end{array}$ & $\begin{array}{l}0.259 \\
(18.5)\end{array}$ & $\begin{array}{l}0.275 \\
(25.8)\end{array}$ & $\begin{array}{c}0.283^{k b l B} \\
(29.4)\end{array}$ & $\begin{array}{c}0.277^{l B} \\
(26.7)\end{array}$ & $\begin{array}{c}0.284^{k b l r B} \\
(30.1)\end{array}$ & $\begin{array}{c}0.277^{I B} \\
(26.8)\end{array}$ \\
\hline & $\mathrm{P} @ 10$ & 0.481 & $\begin{array}{l}0.537 \\
(11.8)\end{array}$ & $\begin{array}{l}0.531 \\
(10.4)\end{array}$ & $\begin{array}{l}0.530 \\
(10.3)\end{array}$ & $\begin{array}{l}0.530 \\
(10.3)\end{array}$ & $\begin{array}{l}0.568 \\
(18.2)\end{array}$ & $\begin{array}{l}0.545 \\
(13.3)\end{array}$ & $\begin{array}{l}0.565 \\
(17.5)\end{array}$ & $\begin{array}{l}0.543 \\
(13.0)\end{array}$ \\
\hline & \#rel_ret & 16536 & 18299 & 18227 & 17603 & 18572 & 18853 & 18690 & 18884 & 18733 \\
\hline & & & (10.7) & $(10.2)$ & $(6.5)$ & $(12.3)$ & (14.0) & $(13.0)$ & (14.2) & (13.3) \\
\hline & robustness & 0 & 0.353 & 0.280 & 0.180 & 0.320 & 0.427 & 0.387 & 0.373 & 0.327 \\
\hline \multirow[t]{4}{*}{ TREC4 } & MAP & 0.217 & $\begin{array}{l}0.261 \\
(20.2)\end{array}$ & $\begin{array}{l}0.256 \\
(17.8)\end{array}$ & $\begin{array}{l}0.238 \\
(9.6)\end{array}$ & $\begin{array}{l}0.243 \\
(12.2)\end{array}$ & $\begin{array}{c}0.279^{k b l r B} \\
(28.7)\end{array}$ & $\begin{array}{c}0.265^{\operatorname{lr} B} \\
(22.3)\end{array}$ & $\begin{array}{c}0.279^{k b l r B} \\
(28.7)\end{array}$ & $\begin{array}{c}0.263^{\operatorname{lr} B} \\
(21.2)\end{array}$ \\
\hline & $\mathrm{P} @ 10$ & 0.461 & $\begin{array}{l}0.455 \\
(-1.3)\end{array}$ & $\begin{array}{l}0.441 \\
(-4.4)\end{array}$ & $\begin{array}{c}0.490 \\
(6.2)\end{array}$ & $\begin{array}{l}0.449 \\
(-2.6)\end{array}$ & $\begin{array}{c}0.498 \\
(8.0)\end{array}$ & $\begin{array}{l}0.480 \\
(4.0)\end{array}$ & $\begin{array}{c}0.492 \\
(6.6)\end{array}$ & $\begin{array}{c}0.469 \\
(1.8)\end{array}$ \\
\hline & \#rel_ret & 3482 & $\begin{array}{l}3815 \\
(9.6)\end{array}$ & $\begin{array}{c}3854 \\
(10.7)\end{array}$ & $\begin{array}{l}3669 \\
(5.4)\end{array}$ & $\begin{array}{l}3621 \\
(4.0)\end{array}$ & $\begin{array}{r}3882 \\
(11.5)\end{array}$ & $\begin{array}{l}3781 \\
(8.6)\end{array}$ & $\begin{array}{c}3947 \\
(13.4)\end{array}$ & $\begin{array}{c}3829 \\
(10.0)\end{array}$ \\
\hline & robustness & 0 & 0.184 & 0.163 & 0.265 & 0.041 & 0.245 & 0.306 & 0.224 & 0.286 \\
\hline \multirow[t]{4}{*}{ TREC5 } & MAP & 0.157 & $\begin{array}{c}0.168 \\
(6.9)\end{array}$ & $\begin{array}{c}0.166 \\
(5.4)\end{array}$ & $\begin{array}{l}0.154 \\
(-2.2)\end{array}$ & $\begin{array}{l}0.174 \\
(10.8)\end{array}$ & $\begin{array}{c}0.171^{b} \\
(9.0)\end{array}$ & $\begin{array}{c}0.172^{k b} \\
(9.2)\end{array}$ & $\begin{array}{c}0.170 \\
(8.0)\end{array}$ & $\begin{array}{c}0.173^{k b} \\
(9.9)\end{array}$ \\
\hline & P@10 & 0.286 & $\begin{array}{l}0.268 \\
(-6.3)\end{array}$ & $\begin{array}{c}0.248 \\
(-13.3)\end{array}$ & $\begin{array}{c}0.240 \\
(-16.1)\end{array}$ & $\begin{array}{c}0.300 \\
(4.9)\end{array}$ & $\begin{array}{l}0.274 \\
(-4.2)\end{array}$ & $\begin{array}{l}0.280 \\
(-2.1)\end{array}$ & $\begin{array}{l}0.268 \\
(-6.3)\end{array}$ & $\begin{array}{l}0.278 \\
(-2.8)\end{array}$ \\
\hline & \#rel_ret & 1936 & $\begin{array}{l}2184 \\
(12.8)\end{array}$ & $\begin{array}{l}2194 \\
(13.3)\end{array}$ & $\begin{array}{l}2055 \\
(6.1)\end{array}$ & $\begin{array}{l}2185 \\
(12.9)\end{array}$ & $\begin{array}{l}2218 \\
(14.6)\end{array}$ & $\begin{array}{l}2166 \\
(11.9)\end{array}$ & $\begin{array}{l}2244 \\
(15.9)\end{array}$ & $\begin{array}{l}2196 \\
(13.4)\end{array}$ \\
\hline & robustness & 0 & 0.040 & -0.060 & -0.080 & 0.120 & 0.160 & 0.160 & 0.080 & 0.080 \\
\hline \multirow[t]{5}{*}{ TREC678 } & MAP & 0.218 & $\begin{array}{l}0.257 \\
(18.0)\end{array}$ & $\begin{array}{l}0.255 \\
(16.8)\end{array}$ & $\begin{array}{l}0.252 \\
(15.5)\end{array}$ & $\begin{array}{l}0.260 \\
(19.3)\end{array}$ & $\begin{array}{c}0.266^{k b l B} \\
(22.0)\end{array}$ & $\begin{array}{c}0.260^{B} \\
(19.2)\end{array}$ & $\begin{array}{c}0.266^{k b l B} \\
(21.8)\end{array}$ & $\begin{array}{c}0.260^{B} \\
(19.0)\end{array}$ \\
\hline & $\mathrm{P} @ 10$ & 0.431 & $\begin{array}{l}0.438 \\
(1.6)\end{array}$ & $\begin{array}{l}0.427 \\
(-0.9)\end{array}$ & $\begin{array}{l}0.420 \\
(-2.6)\end{array}$ & $\begin{array}{l}0.440 \\
(2.0)\end{array}$ & $\begin{array}{c}0.441 \\
(2.2)\end{array}$ & $\begin{array}{c}0.431 \\
(0.0)\end{array}$ & $\begin{array}{c}0.431 \\
(0.0)\end{array}$ & $\begin{array}{l}0.421 \\
(-2.3)\end{array}$ \\
\hline & \#rel_ret & 7287 & 8556 & 8529 & 8192 & 8408 & 8567 & 8552 & 8582 & 8542 \\
\hline & & & (17.4) & $(17.0)$ & (12.4) & (15.4) & (17.6) & (17.4) & (17.8) & (17.2) \\
\hline & robustness & 0 & 0.207 & 0.193 & 0.073 & 0.353 & 0.313 & 0.287 & 0.260 & 0.233 \\
\hline \multirow[t]{4}{*}{ ROBnew } & MAP & 0.278 & $\begin{array}{l}0.312 \\
(12.2)\end{array}$ & $\begin{array}{l}0.307 \\
(10.3)\end{array}$ & $\begin{array}{l}0.330 \\
(18.7)\end{array}$ & $\begin{array}{l}0.343 \\
(23.4)\end{array}$ & $\begin{array}{c}0.326^{k b B} \\
(17.2)\end{array}$ & $\begin{array}{c}0.322^{k b B} \\
(15.9)\end{array}$ & $\begin{array}{c}0.324^{k b B} \\
(16.5)\end{array}$ & $\begin{array}{c}0.321^{k b B} \\
(15.7)\end{array}$ \\
\hline & $\mathrm{P} @ 10$ & 0.421 & $\begin{array}{l}0.405 \\
(-3.8)\end{array}$ & $\begin{array}{l}0.394 \\
(-6.5)\end{array}$ & $\begin{array}{c}0.457 \\
(8.4)\end{array}$ & $\begin{array}{c}0.459 \\
(8.9)\end{array}$ & $\begin{array}{c}0.438 \\
(4.1)\end{array}$ & $\begin{array}{c}0.424 \\
(0.7)\end{array}$ & $\begin{array}{c}0.431 \\
(2.4)\end{array}$ & $\begin{array}{l}0.416 \\
(-1.2)\end{array}$ \\
\hline & \#rel_ret & 2887 & $\begin{array}{l}3172 \\
(9.9)\end{array}$ & $\begin{array}{c}3178 \\
(10.1)\end{array}$ & $\begin{array}{c}3034 \\
(5.1)\end{array}$ & $\begin{array}{r}3213 \\
(11.3)\end{array}$ & $\begin{array}{l}3173 \\
(9.9)\end{array}$ & $\begin{array}{l}3160 \\
(9.5)\end{array}$ & $\begin{array}{c}3180 \\
(10.1)\end{array}$ & $\begin{array}{l}3168 \\
(9.7)\end{array}$ \\
\hline & robustness & 0 & 0.152 & 0.101 & 0.162 & 0.303 & 0.192 & 0.222 & 0.202 & 0.202 \\
\hline \multirow[t]{6}{*}{ TREC910 } & MAP & 0.195 & $\begin{array}{l}0.193 \\
(-1.1)\end{array}$ & $\begin{array}{l}0.189 \\
(-3.3)\end{array}$ & $\begin{array}{c}0.175 \\
(-10.3)\end{array}$ & $\begin{array}{l}0.223 \\
(14.1)\end{array}$ & $\begin{array}{c}0.204^{k b l} \\
(4.7)\end{array}$ & $\begin{array}{c}0.210^{k b l} \\
(7.4)\end{array}$ & $\begin{array}{c}0.203^{k b l} \\
(3.7)\end{array}$ & $\begin{array}{c}0.209^{k b l} \\
(7.3)\end{array}$ \\
\hline & P@10 & 0.307 & $\begin{array}{l}0.293 \\
(-46)\end{array}$ & 0.284 & 0.293 & 0.329 & 0.313 & 0.309 & 0.300 & 0.294 \\
\hline & & & $(-4.6)$ & $(-7.6)$ & $(-4.6)$ & $(7.0)$ & $(2.0)$ & $(0.7)$ & $(-2.3)$ & $(-4.3)$ \\
\hline & \#rel_ret & 3770 & 3987 & 3974 & 3651 & 4001 & 4021 & 3992 & 4025 & 3992 \\
\hline & & & (5.8) & (5.4) & $(-3.2)$ & $(6.1)$ & (6.7) & (5.9) & (6.8) & (5.9) \\
\hline & robustness & 0 & -0.010 & -0.082 & -0.224 & 0.347 & 0.061 & 0.071 & 0 & 0.041 \\
\hline \multirow[t]{4}{*}{ GOV2 } & MAP & 0.270 & $\begin{array}{l}0.290 \\
(7.7)\end{array}$ & $\begin{array}{l}0.290 \\
(7.6)\end{array}$ & $\begin{array}{c}0.222 \\
(-17.6)\end{array}$ & $\begin{array}{l}0.321 \\
(19.1)\end{array}$ & $\begin{array}{c}0.294^{k l B} \\
(9.1)\end{array}$ & $\begin{array}{c}0.294^{k l B} \\
(8.9)\end{array}$ & $\begin{array}{c}0.299^{k b l B} \\
(11.0)\end{array}$ & $\begin{array}{c}0.300^{k b l B} \\
(11.4)\end{array}$ \\
\hline & $\mathrm{P} @ 10$ & 0.533 & $\begin{array}{l}0.568 \\
(6.5)\end{array}$ & $\begin{array}{l}0.560 \\
(5.2)\end{array}$ & $\begin{array}{l}0.544 \\
(2.0)\end{array}$ & $\begin{array}{l}0.604 \\
(13.3)\end{array}$ & $\begin{array}{c}0.577 \\
(8.3)\end{array}$ & $\begin{array}{c}0.574 \\
(7.7)\end{array}$ & $\begin{array}{c}0.585 \\
(9.7)\end{array}$ & $\begin{array}{l}0.588 \\
(10.3)\end{array}$ \\
\hline & \#rel_ret & 17083 & $\begin{array}{l}16994 \\
(-0.5)\end{array}$ & $\begin{array}{l}16939 \\
(-0.8)\end{array}$ & $\begin{array}{l}13534 \\
(-20.8)\end{array}$ & $\begin{array}{c}18036 \\
(5.6)\end{array}$ & $\begin{array}{c}17056 \\
(-0.2)\end{array}$ & $\begin{array}{l}17063 \\
(-0.1)\end{array}$ & $\begin{array}{c}17145 \\
(0.4)\end{array}$ & $\begin{array}{c}17173 \\
(0.5)\end{array}$ \\
\hline & robustness & 0 & 0.094 & 0.060 & -0.309 & 0.456 & 0.114 & 0.101 & 0.121 & 0.148 \\
\hline
\end{tabular}

Table 4: Improvements on different datasets obtained by combining association based and distribution based QE methods.

combinations KLDLCA and Bo1LCA perform better than the baseline as well as KLD. In general, the combination also seems to be safer, in the sense that combination-based expansion usually hurts fewer queries than expansion using either KLD or LCAmod. On a related note, a query wise analysis of the TREC678 dataset shows that out of the 150 queries in this collection, there are 59 queries on which KLD outperforms KLDLCA (with an average improvement in MAP of 0.0148), but KLDLCA does better than KLD on 85 queries, and improves MAP by 0.0255 on average. Similarly, LCAmod performs better than KLDLCA on 68 queries (average improvement in MAP $=0.0360$ ), whereas KLDLCA wins on 81 queries (average improvement in MAP $=0.0594$ ).

Comparing the combination methods with RM3, we find that the combinations are either better than or comparable to RM3 on the older collections, but on the newer collections, RM3 remains the best method by a comfortable margin. We will have to look more carefully into the reasons for this difference in the relative performance of these methods across collections.

Reverse combination As discussed in Section 3.2, we used a distribution-based methods as a starting point, and refined the

\begin{tabular}{lccl}
\hline Dataset & KLDLCA & LCAKLD(KLD weight) & LCAKLD(LCA weight) \\
\hline TREC123 & 0.2826 & 0.2823 & $0.2669^{*}$ \\
TREC4 & 0.2793 & 0.2667 & $0.2283^{*}$ \\
TREC5 & 0.1713 & 0.1741 & 0.1484 \\
TREC678 & 0.2660 & 0.2679 & $0.2416^{*}$ \\
ROBnew & 0.3257 & 0.3298 & 0.3178 \\
TREC910 & 0.2045 & 0.2106 & $0.1826^{*}$ \\
\hline \hline & Bo1RM3 & RM3Bo1(Bo1 weight) & RM3Bo1(RM3 weight) \\
\hline TREC123 & 0.2770 & 0.2759 & 0.2839 \\
TREC4 & 0.2631 & 0.2573 & 0.2690 \\
TREC5 & 0.1727 & 0.1804 & 0.1803 \\
TREC678 & 0.2595 & 0.2562 & $0.2488^{*}$ \\
ROBnew & 0.3214 & 0.3305 & $0.3431^{*}$ \\
TREC910 & 0.2095 & 0.2084 & $0.2138^{*}$ \\
\hline
\end{tabular}

Table 5: Comparing the combination method with its reverse combination method.

set of terms selected using association information. We also tried reversing the order in which these methods were combined, i.e., we first selected terms using an association-based method and then refined this set using distribution information. The final weight of the expansion terms was computed using the distribution-based method. The parameters remained the same as before. From Table 5 ( * denotes a fall that is statistically significant.), we can see that the differences in MAP between the original and the reverse 
combination are small, and not statistically significant. Thus, the combination of two sources of information seems to be useful; the order in which the combination is done does not seem to matter. Finally, we tried the reverse combination with the final weight of the expansion terms being computed using the association-based method. For LCA, this results in a drop in performance that is often significant. However, with RM3 weights, performance generally improves, especially for the new collections. This is consistent with the pattern in Table4.

\section{CONCLUSION}

In this study, our objective was to combine distribution based and association based query expansion methods. Using a number of standard test collections, we have shown that distribution based $\mathrm{QE}$ can be improved by using an association based method to refine term selection. The proposed combination generally gives better results than each individual method, and is comparable to widely used methods such as RM3.

En route to this goal, we also proposed some modifications to a well-known QE methods which lead to improved performance. This may be regarded as an additional contribution of this paper.

In future work, we intend to do a more comprehensive study by investigating other combinations of $\mathrm{QE}$ methods.

\section{REFERENCES}

[1] Nasreen Abdul-Jaleel, James Allan, W. Bruce Croft, Fernando Diaz, Leah S. Larkey, Xiaoyan Li, Mark D. Smucker, and Courtney Wade. 2004. UMass at TREC 2004 Novelty and HARD. In TREC.

[2] G. Amati. 2003. Probability Models for Information Retrieval Based on Divergence from Randomness. University of Glasgow.

[3] Gianni Amati and Cornelis Joost Van Rijsbergen. 2002. Probabilistic models of information retrieval based on measuring the divergence from randomness. ACM Trans. Inf. Syst. 20 (October 2002), 357-389. Issue 4.

[4] Michael Bendersky, Donald Metzler, and W. Bruce Croft. 2011. Parameterized concept weighting in verbose queries. In Proceedings of the 34th international ACM SIGIR Conference on Research and Development in Information Retrieval (SIGIR '11). ACM, New York, NY, USA, 605-614.

[5] J. Bhogal, A. Macfarlane, and P. Smith. 2007. A review of ontology based query expansion. Inf. Process. Manage. 43, 4 (July 2007), 866-886.

[6] Bodo Billerbeck and Justin Zobel. 2004. Questioning query expansion: an examination of behaviour and parameters. In ADC '04: Proceedings of the 15th Australasian database conference. Australian Computer Society, Inc., Darlinghurst, Australia, Australia, 69-76.

[7] Guihong Cao, Jian-Yun Nie, Jianfeng Gao, and Stephen Robertson. 2008. Selecting good expansion terms for pseudo-relevance feedback. In Proceedings of the 31st Annual International ACM SIGIR Conference on Research and Development in Information Retrieval (SIGIR '08). ACM, New York, NY, USA, 243-250.

[8] Claudio Carpineto, Renato de Mori, Giovanni Romano, and Brigitte Bigi. 2001 An information-theoretic approach to automatic query expansion. ACM Trans. Inf. Syst. 19, 1 (2001), 1-27.

[9] Claudio Carpineto and Giovanni Romano. 2012. A Survey of Automatic Query Expansion in Information Retrieval. ACM Comput. Surv. 44, 1, Article 1 (Jan. 2012), 50 pages.

[10] Claudio Carpineto, Giovanni Romano, and Vittorio Giannini. 2002. Improving retrieval feedback with multiple term-ranking function combination. ACM Trans. Inf. Syst. 20, 3 (2002), 259-290.

[11] Kevyn Collins-Thompson and Jamie Callan. 2007. Estimation and use of uncertainty in pseudo-relevance feedback. In Proceedings of the 30th Annual International ACM SIGIR Conference on Research and Development in Information Retrieval (SIGIR '07). ACM, New York, NY, USA, 303-310.

[12] Tamas E. Doszkocs. 1978. An Associative Interactive Dictionary for Online Bibliographic Searching. In Jerusalem Conference on Information Technology, 1978 489-492.

[13] Hui Fang. 2008. A Re-examination of Query Expansion Using Lexical Resources. In In Proceedings of ACL-08: HLT. 139-147.

[14] Y. Jing and W.B. Croft. 1994. An association thesaurus for information retrieval. In Proceedings of the Intelligent Multimedia Information Retrieval Systems (RIAO '94, New York, NY), 1994. 146-160.
[15] K. Sparck Jones, S. Walker, and S.E. Robertson. 2000. A probabilistic model of information retrieval: development and comparative experiments: Part 1. Information Processing \& Management 36, 6 (2000), 779-808.

[16] Victor Lavrenko and W. Bruce Croft. 2001. Relevance-Based Language Models. In SIGIR, 2001. 120-127.

[17] Yuanhua Lv and ChengXiang Zhai. 2009. A comparative study of methods for estimating query language models with pseudo feedback. In Proceedings of the 18th ACM conference on Information and knowledge management (CIKM '09). ACM, New York, NY, USA, 1895-1898.

[18] Yuanhua Lv, ChengXiang Zhai, and Wan Chen. 2011. A boosting approach to improving pseudo-relevance feedback. In Proceedings of the 34th international ACM SIGIR Conference on Research and Development in Information Retrieval (SIGIR '11). ACM, New York, NY, USA, 165-174.

[19] Craig Macdonald, Ben He, Vassilis Plachouras, and Iadh Ounis. 2005. University of Glasgow at TREC 2005: Experiments in Terabyte and Enterprise Tracks with Terrier. In TREC, Ellen M. Voorhees and Lori P. Buckland (Eds.), Vol. Special Publication 500-266. National Institute of Standards and Technology (NIST).

[20] Rila Mandala, Takenobu Tokunaga, and Hozumi Tanaka. 1999. Combining Multiple Evidence from Different Types of Thesaurus for Query Expansion. (1999).

[21] José R. Pérez-Agüera and Lourdes Araujo. 2008. Comparing and Combining Methods for Automatic Query Expansion. CoRR abs/0804.2057 (2008).

[22] Vassilis Plachouras, Ben He, and Iadh Ounis. 2004. University of Glasgow at TREC 2004: Experiments in Web, Robust, and Terabyte Tracks with Terrier. In TREC, Ellen M. Voorhees and Lori P. Buckland (Eds.), Vol. Special Publication 500-261. National Institute of Standards and Technology (NIST).

[23] Yonggang Qiu and Hans-Peter Frei. 1993. Concept based query expansion. In Proceedings of the 16th Annual International ACM SIGIR Conference on Research and Development in Information Retrieval (SIGIR '93). ACM, New York, NY, USA, $160-169$

[24] Stephen Robertson. 2004. Understanding inverse document frequency: On theoretical arguments for IDF. Fournal of Documentation 60 (2004), 2004

[25] S. E. Robertson. 1991. On term selection for query expansion. J. Doc. 46, 4 (Jan. 1991), 359-364.

[26] Gerard Salton (Ed.). 1971. The SMART Retrieval System - Experiments in Automatic Document Processing. Prentice Hall, Englewood, Cliffs, New Jersey.

[27] Renxu Sun, Chai-Huat Ong, and Tat-Seng Chua. 2006. Mining dependency relations for query expansion in passage retrieval. In Proceedings of the 29th Annual International ACM SIGIR Conference on Research and Development in Information Retrieval (SIGIR '06). ACM, New York, NY, USA, 382-389.

[28] Olga Vechtomova, Stephen Robertson, and Susan Jones. 2003. Query Expansion with Long-Span Collocates. Inf. Retr. 6, 2 (April 2003), 251-273.

[29] H. C. Wu, R. W. P. Luk, K. F. Wong, and J. Y. Nie. 2012. A split-list approach for relevance feedback in information retrieval. Inf. Process. Manage. 48, 5 (Sept. 2012), 969-977.

[30] Jinxi Xu and W. Bruce Croft. 1996. Query Expansion Using Local and Global Document Analysis. In Proceedings of the 19th Annual International ACM SIGIR Conference on Research and Development in Information Retrieval (SIGIR '96). ACM, New York, NY, USA, 4-11.

[31] Jinxi Xu and W. Bruce Croft. 2000. Improving the effectiveness of information retrieval with local context analysis. ACM Trans. Inf. Syst. 18, 1 (2000), 79-112.

[32] Yang Xu, Gareth J.F. Jones, and Bin Wang. 2009. Query dependent pseudorelevance feedback based on wikipedia. In Proceedings of the 32nd international ACM SIGIR Conference on Research and Development in Information Retrieval (SIGIR '09). ACM, New York, NY, USA, 59-66.

[33] Xiaobing Xue and W. Bruce Croft. 2013. Modeling reformulation using query distributions. ACM Trans. Inf. Syst. 31, 2, Article 6 (May 2013), 34 pages.

[34] Chengxiang Zhai and John Lafferty. 2001. Model-based Feedback in the Language Modeling Approach to Information Retrieval. In Proceedings of the Tenth International Conference on Information and Knowledge Management (CIKM '01). ACM, New York, NY, USA, 403-410. https://doi.org/10.1145/502585.502654

\section{APPENDIX}

\section{A LCAMOD: A MODIFIED FORM OF LCA}

LCA [31] is one of the most well-known association based term selection methods. In this appendix, we first review the original LCA formula as proposed by Xu and Croft [31]. Next, we introduce some simple modifications in the basic formula for LCA in order to improve its performance. We also present experimental results to confirm that our modifications actually result in better retrieval effectiveness.

In LCA, all terms from the top ranked set are considered as candidate expansion terms. Equations 17 through 20 show how 
co-occurrence is calculated for a candidate term $t$ and a query $Q$ consisting of terms $q_{1}, \ldots, q_{k}$ (recall that $N_{t}$ has its obvious meaning, $P R D$ denotes the set of pseudo-relevant documents, and $\delta$ is set to 0.1 as suggested by $\mathrm{Xu}$ and Croft [31]).

$$
\begin{gathered}
i d f_{t}=\min \left(\log _{10}\left(N / N_{t}\right) / 5.0,1.0\right) \\
\operatorname{co}\left(t, q_{i}\right)=\sum_{d \in P R D} t f(t, d) * t f\left(q_{i}, d\right) \\
\text { codegree }\left(t, q_{i}\right)=\frac{\log _{10}\left(\operatorname{co}\left(t, q_{i}\right)+1\right) * i d f_{t}}{\log _{10}|P R D|} \\
S(t)=\sum_{i=1}^{k} i d f_{q_{i}} * \log _{10}\left(\delta+\operatorname{codegree}\left(t, q_{i}\right)\right)
\end{gathered}
$$

The $T$ terms with the highest $S(t)$ scores are selected as expansion terms. Finally, the $j$-th "best" term is weighted according to Equation 21.

$$
\text { score }_{\exp }(t)=1.0-\frac{0.9 * j}{T}
$$

We did not use noun-phrases or passage level retrieval, since the authors show that these refinements do not have much impact. Our experiments confirm that our implementation yields very similar results for the collections and settings mentioned in [31].

\section{A.1 Modified LCA}

Our implementation of the above formulae did not yield the expected improvements across collections. A failure analysis suggests that Equation 18 might be the culprit. For example, consider the TREC4 query: "How has affirmative action affected the construction industry?". Two terms papuc (Pennsylvania Public Utility Commission) and limerick are very highly ranked among candidate terms by Equation 20, even though these are not useful expansion terms. This is because in one top document, the word 'papuc' occurs 21 times and a query word ('construction') occurs 35 times. The multiplication of raw term frequencies in Equation 18 results in a very high weight for the term 'papuc'. A similar problem occurs in case of 'limerick', which occurs 17 times in one document.

Our hypothesis is that the number of co-occurrences of a term pair can only be as large as the minimum term frequency of the two terms under consideration. We also hypothesize that co-occurrences in a document are more important if the document is "close" (or similar) to the query. Finally, we use the $i d f$ factor $^{5}$ for a candidate expansion term when calculating its co-occurrence (Equation 10); it is no longer used when calculating co-degree (Equation 11). Equations 10 through (12) in Section 3.1.3 summarise these changes.

\section{A.2 Experimental results}

We compare the effectiveness of LCAmod against the following baselines. A * denotes an improvement (over original formula) that is statistically significant.

(1) No feedback. The original, unexpanded queries are used for retrieval using the baseline method described in Section 4.

(2) LCA. To determine the parameters for LCA, we used the TREC678 collection as a "tuning" dataset, as TREC678 contains a large set of queries. We varied the number of top-ranked documents $(D)$ from 10 to 50 in steps of 10 , while the number of expansion terms $(T)$ was

\footnotetext{
${ }^{5}$ Note that we use Robertson's idf formula [24] (Equation 9) instead of Equation 17.
}

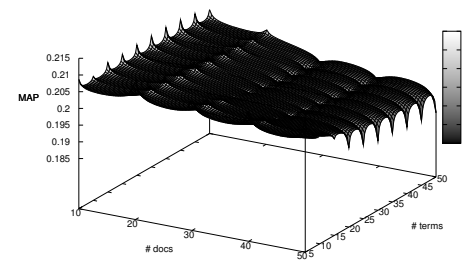

Figure 2: Performance (MAP) of LCA on TREC678 for different parameter. settings.

\begin{tabular}{ccccc}
\hline Dataset & Measure & Baseline & LCA & LCAmod \\
\hline TREC123 & MAP & 0.218 & $0.213(-2.4)$ & $0.259^{*}(18.5)$ \\
& P@10 & 0.481 & $0.472(-1.8)$ & $0.530(10.3)$ \\
& \#rel_ret & 16536 & $15714(-5.0)$ & $17603(6.5)$ \\
& robustness & 0 & 35 & 54 \\
\hline TREC4 & MAP & 0.217 & $0.219(1.1)$ & $0.238(9.6)$ \\
& P@10 & 0.461 & $0.400(-13.3)$ & $0.490(6.2)$ \\
& \#rel_ret & 3482 & $3507(0.7)$ & $3669(5.4)$ \\
& robustness & 0 & 38 & 53 \\
\hline TREC5 & MAP & 0.157 & $0.130(-17.6)$ & $0.154^{*}(-2.2)$ \\
& P@10 & 0.286 & $0.210(-26.6)$ & $0.240(-16.1)$ \\
& \#rel_ret & 1936 & $1894(-2.2)$ & $2055(6.1)$ \\
& robustness & 0 & 20 & 38 \\
\hline TREC678 & MAP & 0.218 & $0.209(-4.2)$ & $0.252^{*}(15.5)$ \\
& P@10 & 0.431 & $0.379(-12.2)$ & $0.420(-2.6)$ \\
& \#rel_ret & 7287 & $7367(1.1)$ & $8192(12.4)$ \\
& robustness & 0 & 36 & 50 \\
\hline ROBnew & MAP & 0.278 & $0.264(-5.0)$ & $0.330^{*}(18.7)$ \\
& P@10 & 0.421 & $0.385(-8.6)$ & $0.457(8.4)$ \\
& \#rel_ret & 2887 & $2864(-0.8)$ & $3034(5.1)$ \\
& robustness & 0 & 36 & 53 \\
\hline TREC910 & MAP & 0.195 & $0.155(-20.6)$ & $0.175^{*}(-10.3)$ \\
& P@10 & 0.307 & $0.231(-24.9)$ & $0.293(-4.6)$ \\
& \#rel_ret & 3770 & $3440(-8.8)$ & $3651(-3.2)$ \\
& robustness & 0 & 27 & 33 \\
\hline
\end{tabular}

Table 6: Improvements on different datasets obtained by modifying LCA.

varied from 5 to 50 in steps of 5 . Xu and Croft [31] recommended using $D=70$ and $T=70$. In our setup, however, a setting of $D=10$ documents and $T=40$ expansion terms works well. Figure 2 shows that these settings work well in terms of MAP. We use these values on all collections used in our experiments. A similar exercise suggests that the same settings can be used for LCAmod as well.

LCAmod. Our first goal is to verify that the proposed modifications to the LCA formula actually yield improvements in retrieval performance. Table 6 shows that, with 'title only' queries, our implementation of the original LCA formula results in a drop in MAP for almost all collections. Only for the TREC4 collection (in which queries consist of a description only), a marginal improvement is observed, suggesting that the original method works better for longer queries. Indeed, the experiments by $\mathrm{Xu}$ and $\mathrm{Croft}$ all used relatively long queries, e.g., the full TREC3 queries (including title, description and narrative fields), the TREC4 queries, and the description field of TREC 5 queries.

Compared to the original formula, the modified formula results in significant improvements in MAP across all data sets. On the ROBnew collection in particular, it performs very well, outperforming the original method by nearly $24 \%$. For the TREC 4 corpus, an improvement of about $11.41 \%$ (over LCA) is observed. The modifications thus seem to be effective for both short as well as relatively longer queries. The LCAmod method is also better in terms of P@10, number of relevant documents retrieved, and robustness. Based on Table 6, we conclude that LCAmod is more effective, and can be used in place of LCA. 